\title{
Scaling for the Discrete Mathieu Equation
}

\author{
D. J. Thouless
}

Department of Physics FM-15, University of Washington, Seattle, WA 98195 USA*, and Cavendish Laboratory, Cambridge, England

\begin{abstract}
The scaling of the total width of the band for the discrete Mathieu equation is studied in the critical region near the transition between localized and extended states, for the special case in which there is one period of the modulation for $p$ lattice spacings. A general expression for the bandwidth $W$ in the critical region is found. At the critical point an analytic expression for $p W$ is found which agrees to one part in $10^{8}$ with the result deduced from numerical work.
\end{abstract}

\section{Introduction}

In an earlier paper [1], which I refer to as BW, a study was made of the total band width for the discrete Mathieu equation, which can be written as

$$
V_{1} a_{n-1}+2 V_{2} \cos \left(2 \pi n \phi+k_{2}\right) a_{n}+V_{1} a_{n+1}=E a_{n}
$$

to simplify the subsequent discussion I take $V_{1}$ and $V_{2}$ to be positive. From the work of Aubry and Andre [2] it was already known that this equation has a critical point for $V_{1}=V_{2}$, where, in the incommensurate limit with $\phi$ irrational, all eigenfunctions become extended instead of localized, and the total bandwidth, again in the incommensurate limit, appears to be equal to $4\left|V_{2}-V_{1}\right|$, so that the bandwidth vanishes at the critical point. In BW I gave a derivation of this linear behaviour of the bandwidth, and did some numerical studies of the way the bandwidth behaves when the common period, $p$, of the sinusoidal term and the lattice becomes large. In this commensurate case, with

$$
\phi=q / p,
$$

the bandwidth is defined as the measure of the union over all values of the phase $k_{2}$ of the spectrum of Eq. 1, which consists of a set of $p$ bands which are generally distinct, except at $E=0$, where there are two bands touching for even values of $p$. I found what appeared to be a scaling behavior of the bandwidth in the critical

\footnotetext{
^ Permanent address
} 
region; the deviation of the bandwidth from $4\left|V_{2}-V_{1}\right|$ is proportional to $\left(V_{1} / V_{2}\right)^{p / 2} / p$ for $V_{1}<V_{2}$, and at the critical point $V_{1}=V_{2}$ the bandwidths converged to $9.3300 p^{-1}$. This limit appeared to be independent of the numerator $q$, although the rate of approach to the limit depended strongly on $q$. The limit was approached from above for even $p$ and from below for odd $p$. An analogy was drawn with finite size scaling theory of critical phenomena $[3,4]$, but no argument for this independence of $q$ was presented, and no explanation of the numerical value of the coefficient at the critical point was given.

In this paper I present a detailed study of the special case $q=1$, which happens to be the value of $q$ for which the convergence of the bandwidth to its limit is slowest, so slow that some uncertainty was expressed in BW as to whether there was actually a common limit for odd and even values of $p$ in that particular case. I find that the bandwidth can be expressed in terms of an integral over the Green functions (resolvents) of some finite matrices. For $p$ large this can be approximated by the Green function for a continuum problem, which is essentially the differential equation satisfied by parabolic cylinder functions. This allows me to express the integrand in terms of gamma functions, and the gamma functions can be manipulated to obtain an expression, valid for large $p$ and finite $p\left(V_{2}-V_{1}\right)$, which is an integral over a trigonometric function. This therefore gives a complete description of the scaling behavior of the bandwidth for $q=1$ in the critical region. At the critical point $V_{1}=V_{2}$ the integral can be expressed in terms of Catalan's constant, and its numerical value agrees to eight significant figures with the results I obtained by further numerical work along the lines reported in BW. As a preface to this analytical work I give a brief summary of some of the new numerical work.

\section{Summary of Numerical Results}

The positions of the band edges were obtained from Eqs. (2.3) and (2.4) of BW for odd $p$ and the corresponding equations for even $p$ by using a NAG library subroutine for finding the eigenvalues of a tridiagonal real symmetric matrix. The complete set of band edges was found in this way, and they were then arranged in ascending order so that band widths and band gaps could be found from the differences between successive eigenvalues. For $V_{1}=V_{2}$ the product $p W$ of the bandwidth $W$ with $p$ was studied in some detail, but no major differences were discovered from the results reported in BW. In all cases the values of $p W$ for odd $p$ were below those for even $p$. The convergence to a common limit was convincing for all sequences that I examined except for the case $q=1$. The convergence was particularly rapid for the cases $q=2$ with $p$ odd and $q=p / 2-1$ with $p$ even, and there the deviation from the limiting value seemed to go like $p^{-2}$; it was also rapid for even $p$ and $q$ close to $p / 4$. For $q=1$ the deviation from the common limit of the other sequences seemed to decrease slower than $p^{-1 / 3}$.

Two sequences I used were $p=249,499,999,1999$ with $q=2$, and $p=248,496$, 992, 1984 with $q=p / 2-1$, which gave the results $9.32989645,9.32993703$, 9.32994593, 9.32994821 and 9.33004952, 9.32997407, 9.32995522, 9.32995058 for $p W / V_{1}$. Each of these sequences extrapolates to 9.3299490. Extrapolations I made on other sequences of the same sort agree with this to one part in $10^{8}$. 


\section{Evaluation of the Scaling Function}

For odd values of $p$ the band edges are determined by the eigenvalues $E^{++}, E^{--}$of Eqs. (3.1) and (3.2) of BW. These have the form

$$
V_{1} c_{n-1}+\left[2 V_{2} \cos \frac{2 n q \pi}{p} \pm V_{1} \delta_{n s}\right] c_{n}+V_{1} c_{n+1}=E^{+ \pm} c_{n},
$$

with $0 \leqq n \leqq s=(p-1) / 2$ and $c_{-1}=c_{1}, c_{s+1}=0$, and

$$
V_{1} c_{n-1}+\left[2 V_{2} \cos \frac{2 n q \pi}{p} \pm V_{1} \delta_{n s}\right] c_{n}+V_{1} c_{n+1}=E^{- \pm} c_{n},
$$

with $0<n \leqq s$ and $c_{0}=0, c_{s+1}=0$. These two equations give half of the band edges, and the other half are found by changing the signs of these eigenvalues.

A key observation for the subsequent work is that for $q=1$, and only for $q=1$ (or equivalent values of $q$ such as $p-1$ ), the positive eigenvalues $E^{++}$alternate with the $E^{--}$, and so do the negative eigenvalues, although the order is reversed. Thus the positive $E^{++}$and negative $E^{--}$are at the tops of bands, while the positive $E^{--}$and negative $E^{++}$are at bottoms of bands. This is easy to show for $V_{2} \gg V_{1}$, where a perturbative argument can be used, and Mouche [5] has shown that there are no degeneracies when $V_{1} / V_{2}$ is varied, so this ordering is preserved for $V_{1}=V_{2}$. If the eigenvalues of these equations are numbered in ascending numerical order the widths of the band gaps are given by

$$
\left|E_{n}^{--}\right|-\left|E_{n}^{++}\right| \text {, }
$$

while the highest band edge is at $E_{s+1}^{++}$. The gaps and lowest band edge obtained by changing the signs of these eigenvalues must also be taken into account. The bandwidth can be found by subtracting the sum of band gaps from the separation between the two extreme band edges, and this gives

$$
W=2 \sum_{n=1}^{s+1}\left|E_{n}^{++}\right|-2 \sum_{n=1}^{s}\left|E_{n}^{--}\right| .
$$

This relation can be written in terms of the Green functions for the problem, using two contours that surround the spectrum on the negative and positive real axes, as

$$
W=\frac{2 i}{\pi} \int_{-i \infty}^{i \infty}\left\{\operatorname{tr}\left[G^{++}(z)-G^{--}(z)\right] z-1\right\} d z ;
$$

the constant term is inserted in the integrand because $G^{++}$and $G^{--}$are Green functions for matrices whose orders differ by unity. Since the integrand is of order $z^{-1}$ for large $z$ the limits of integration on the imaginary axis must be taken to infinite symmetrically. It is shown in the appendix that

$$
\begin{gathered}
\operatorname{tr}\left[G^{++}(z)-G^{-+}(z)\right]=-\frac{d}{d z} \ln G_{00}^{++}(z), \\
\operatorname{tr}\left[G^{-+}(z)-G^{--}(z)\right]=-\frac{d}{d z} \ln \left[G_{s s}^{-+}(z) / G_{s s}^{--}(z)\right] .
\end{gathered}
$$


This allows Eq. (7) to be written as

$$
W=\frac{2 i}{\pi} \int_{-i \infty}^{i \infty} \ln \left[z G_{00}^{++}(z) G_{s s}^{-+}(z) / G_{s s}^{--}(z)\right] d z .
$$

The Green functions can be written in terms of the solutions of Eq. (1) for arbitrary $E=z$. If $a_{n}(z)$ is the solution that satisfies $a_{s+1}=a_{s}$, and $b_{n}(z)$ the solution satisfying $b_{0}=0$, then Eq. (9) becomes

$$
W=\frac{2 i}{\pi} \int_{-i \infty}^{i \infty} \ln \frac{z a_{0}\left(b_{s}+b_{s+1}\right)}{V_{1}\left(a_{-1}-a_{1}\right)\left(b_{s+1}-b_{s}\right)} d z .
$$

So far this result is exact. To make further progress for $p$ large I make continuum approximations for $(-1)^{n} a_{n}$ and $b_{n}$, which should be valid in some neighborhood of $n=0$ or $n=s$ for sufficiently small $z$ and $\left|V_{2}-V_{1}\right|$. To do this I replace $2 V_{1} \cosh (d / d n)$ and $2 V_{2} \cos (2 \pi n \phi)$ in Eq. (1) by their quadratic approximations, and the resulting differential equation is then the equation for a parabolic cylinder function. For simplicity in the subsequent work I take an energy scale in which $V_{1} V_{2}=1$. In the notation used by Abramowitz and Stegun [6] the approximations are

$$
\begin{gathered}
a_{n} \approx(-1)^{n} E\left(\frac{p\left(V_{2}-V_{1}\right)}{2 \pi}-\frac{p z}{4 \pi}, \sqrt{\frac{4 \pi}{p V_{1}}} n\right), \\
b_{n} \approx E^{*}\left(\frac{p\left(V_{2}-V_{1}\right)}{2 \pi}+\frac{p z}{4 \pi}, \sqrt{\frac{4 \pi}{p V_{1}}}\left(\frac{1}{2} p-n\right)\right) .
\end{gathered}
$$

From this point onwards the work is just an application of standard results known for special functions. To evaluate Eq. (10) the logarithmic derivative at the origin of the parabolic cylinder functions must be used, and this is given by an expression involving gamma functions [6]. I define new variables by

$$
y=p z / 8 \pi i, \quad v=p\left(V_{2}-V_{1}\right) / 4 \pi,
$$

and then substitution of Eq. (11) into Eq. (10) gives

$$
W \approx \frac{32}{p} \int_{0}^{\infty} \ln \frac{\Gamma\left(\frac{3}{4}+y+i v\right) \Gamma\left(\frac{3}{4}+y-i v\right)}{y \Gamma\left(\frac{1}{4}+y+i v\right) \Gamma\left(\frac{1}{4}+y-i v\right)} d y .
$$

The contributions to the integral of the gamma functions in the numerator and denominator of the logarithm cancel over most of the range, so that this expression can be written as

$$
W=-\frac{32}{p}\left\{\frac{1}{2} \ln (2 \pi)-\int_{\frac{1}{4}+i v}^{\frac{3}{4}+i v} \ln [\Gamma(y) \Gamma(1-y)] d y\right\} .
$$

The argument of the logarithm is equal to $[7] \pi / \sin (\pi y)$, so a complete expression for the scaling behavior is given by

$$
W=\frac{16}{p} \int_{-1 / 4}^{1 / 4} \ln [2(\cos 2 \pi y+\cosh 2 \pi v)] d y .
$$


The scaling variable in this expression is $v=p\left(V_{2}-V_{1}\right) / 4 \pi \sqrt{V_{1} V_{2}}$, so the crossover exponent is unity. For large $v$ Eq. (15) gives

$$
W \sim 16 \pi|v| / p+(32 / \pi p) \exp (-2|v|) .
$$

The first term in this expression is just the result $W \sim 4\left|V_{2}-V_{1}\right|$ which has been known for a long time [2]. The second term is a correction for systems with finite period. This general form was found in BW, but the value of the coefficient was found to be variable and somewhat lower; this is probably because the numerical work was carried out too far from the critical region.

Equation 15 can be more conveniently expressed in terms of an indefinite integral. Differentiation of this equation with respect to $v$ shows that $W$ satisfies the differential equation

$$
d W / d v=(32 / p) \arctan [\sinh (2 \pi v)]
$$

With a little further manipulation the integral of this equation can be written in the form

$$
p W=16 \pi v+\frac{32}{\pi} \int_{0}^{\exp (-2 \pi v)} \arctan t \frac{d t}{t}
$$

This can be expressed in terms of the dilogarithm function of imaginary argument. The function is shown in Fig. 1.

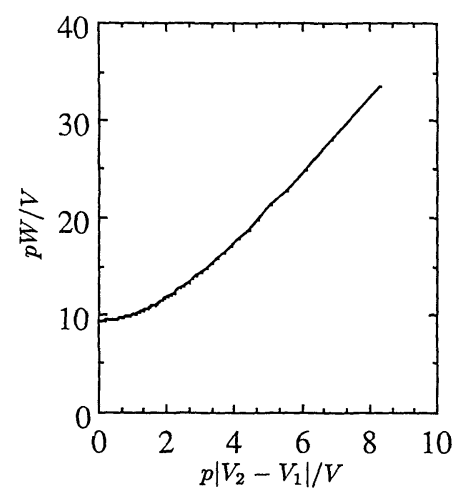

Fig. 1. The scaling function of Eq. (18). The scaled bandwidth $p W / V$ is shown as a function of the scaled distance from the critical point $p\left|V_{2}-V_{1}\right| / V$

For $v=0$ the integral can be reduced to a known result. If the integrand is written as a power series in $t$, then the integral is Catalan's constant $\beta(2)$ [8]. This gives

$$
p W=32 \beta(2) / \pi \approx 9.32994893
$$

in excellent agreement with the numerical results quoted in Sect. 2. 


\section{Discussion}

The precise agreement of the analytical expression for $p W$ at the critical point for $q=1$ with the numerical estimates for this quantity obtained for other values of $q$ is very encouraging for a scaling theory of the transition between localized and extended states in the one-dimensional quasiperiodic system. It shows that there are features of the transition which have some degree of universality. The analytical result suggests that a study of the effect of perturbations on this problem might be practicable, so that one should be able to find a larger class of quasiperiodic problems with the same behavior in the critical region.

I have not made any attempt to assess the validity of the approximations that lead to Eq. (11), except in the formal sense that the terms neglected in the differential equation vanish in the limit of $p$ infinite.

\section{Appendix}

The Green function identities used to go from Eq. (7) to Eq. (8) are derived here. The Green functions $G^{++}$and $G^{-+}$are resolvents for matrices that are of order $s+1$ and $s$ respectively. The matrices differ only in the elements $M_{00}$ and $M_{01}=M_{10}$ that connect the component 0 to the other components. From this follows the result

$$
G_{i j}^{++}-G_{i j}^{-+}=G_{i 0}^{++} M_{01} G_{1 j}^{-+} \text {for } i, j>0,
$$

which has the special case

$$
G_{1 j}^{-+}=G_{1 j}^{++} /\left(1+G_{10}^{++} M_{01}\right) .
$$

Also, because the matrix is tridiagonal, there are relations

$$
\begin{gathered}
M_{01} G_{10}^{++}=\left(z-M_{00}\right) G_{00}^{++}-1, \\
M_{01} G_{1 j}^{++}=\left(z-M_{00}\right) G_{0 j}^{++}, \text {for } j>0 .
\end{gathered}
$$

Substitution of Eqs. (A2) and (A3) in (A1) gives

$$
G_{i i}^{++}-G_{i i}^{-+}=G_{i 0}^{++} G_{0 i}^{++} / G_{00}^{++} .
$$

Since the derivative of the resolvent operator with respect to $z$ is minus the square of the operator, this leads to the result

$$
\operatorname{tr}\left[G^{++}(z)-G^{-+}(z)\right]=-\frac{d}{d z} \ln G_{00}^{++}(z) .
$$

Similar use can be made of the relation

$$
G_{i j}^{-+}-G_{i j}^{-{ }^{-}}=2 V_{1} G_{i s}^{-+} G_{s j}^{--}
$$

and its special case

$$
G_{s j}^{--}=G_{s j}^{-+} /\left(1+2 V_{1} G_{s s}^{-+}\right) .
$$


These give

$$
\operatorname{tr}\left[G^{-+}(z)-G^{--}(z)\right]=-\frac{d}{d z} \ln \left(1+2 V_{1} G_{s s}^{-+}\right) .
$$

Since Eq. (A7) gives

$$
1+2 V_{1} G_{s s}^{-+}=G_{s s}^{-+} / G_{s s}^{--}
$$

the result

$$
\operatorname{tr}\left[G^{-+}(z)-G^{--}(z)\right]=-\frac{d}{d z} \ln \left[G_{s s}^{-+}(z) / G_{s s}^{--}(z)\right]
$$

is obtained.

Acknowledgements. This work was supported in part by the US National Science Foundation under Grant No. 86-13598. I wish to thank Stellan Ostlund and Paul Steinhardt for encouraging my renewed interest in this problem, and Yosi Avron for pointing out the importance of van Mouche's work and for a number of other helpful comments on the paper.

\section{References}

1. Thouless, D.J.: Bandwidths for a quasiperiodic tight-binding model. Phys. Rev. 28, 4272-4276 (1983)

2. Aubry, S., André, G.: Analyticity breaking and Anderson localization in incommensurate lattices. Ann. Israel Phys. Soc. 3, 133-164 (1980)

3. Barber, M.N.: Finite-size scaling. In: Phase transitions and critical phenomena, Vol. 8, pp. 145-266. Domb, C., Lebowitz, J.L. (eds.). London: Academic Press 1983

4. Nightingale, M.: Finite-size scaling and phenomenological renormalization. J. Appl. Phys. 53, $7927-7932$ (1982)

5. v. Mouche, P.: The coexistence problem for the discrete Mathieu operator. Commun. Math. Phys. 122, 23-34 (1989)

6. Abramowitz, M., Stegun, I.A.: Handbook of mathematical functions, pp. 687-693. New York: Dover 1965

7. Abramowitz, M., Stegun, I.A.: Op. cit., p. 256

8. Abramowitz, M., Stegun, I.A.: Op. cit., p. 807

Communicated by B. Simon

Received July 17, 1989 
